\title{
Electronic properties of homoepitaxial (111) highly boron-doped diamond films
}

Haitao Ye, Niall Tumilty, Mose Bevilacqua, Stephane Curat, Milos Nesladek, Bertrand Bazin, Philippe Bergonzo, and Richard B Jackman

Citation: Journal of Applied Physics 103, 054503 (2008); doi: 10.1063/1.2837114

View online: https://doi.org/10.1063/1.2837114

View Table of Contents: http://aip.scitation.org/toc/jap/103/5

Published by the American Institute of Physics

\section{Articles you may be interested in}

Growth and electrical characterisation of $\delta$-doped boron layers on (111) diamond surfaces

Journal of Applied Physics 111, 033710 (2012); 10.1063/1.3682760

High hole mobility in boron doped diamond for power device applications

Applied Physics Letters 94, 092102 (2009); 10.1063/1.3086397

Evaluation of ohmic contacts formed by $\mathrm{B}^{+}$implantation and Ti-Au metallization on diamond Journal of Applied Physics 74, 1179 (1993); 10.1063/1.354918

An insight of p-type to n-type conductivity conversion in oxygen ion-implanted ultrananocrystalline diamond films by impedance spectroscopy

Applied Physics Letters 110, 033102 (2017); 10.1063/1.4974077

Potential barrier heights at metal on oxygen-terminated diamond interfaces

Journal of Applied Physics 118, 204505 (2015); 10.1063/1.4936317

$\mathrm{N}$-type control of single-crystal diamond films by ultra-lightly phosphorus doping

Applied Physics Letters 109, 142102 (2016); 10.1063/1.4964382

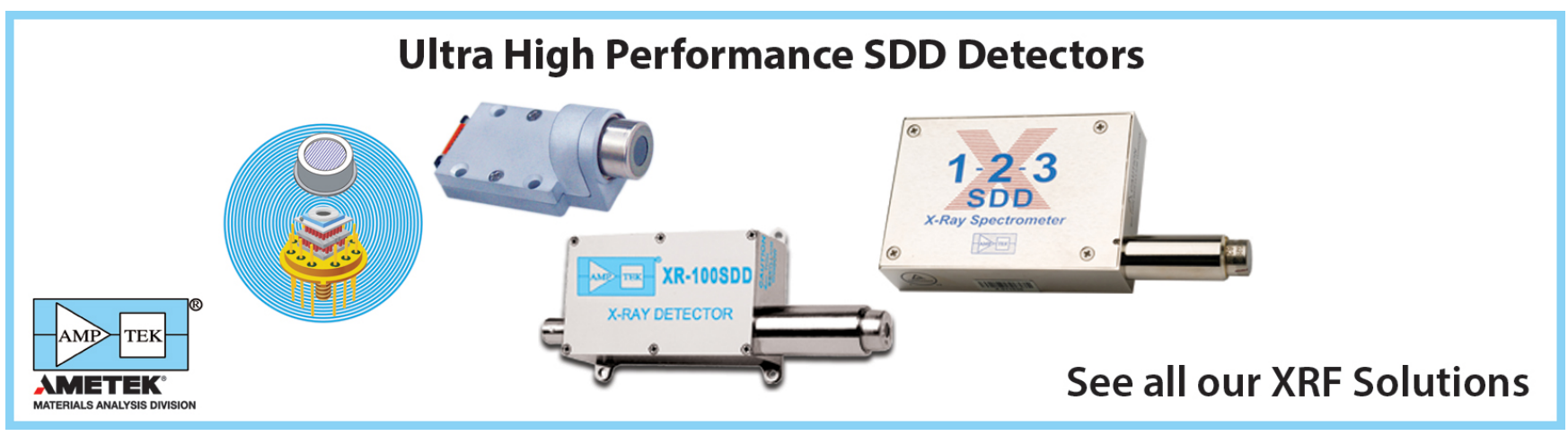




\title{
Electronic properties of homoepitaxial (111) highly boron-doped diamond films
}

\author{
Haitao Ye, ${ }^{1}$ Niall Tumilty, ${ }^{1}$ Mose Bevilacqua, ${ }^{1}$ Stephane Curat, ${ }_{1}^{1}$ Milos Nesladek, ${ }^{2}$ \\ Bertrand Bazin, ${ }^{2}$ Philippe Bergonzo, ${ }^{2}$ and Richard B Jackman ${ }^{1, a)}$ \\ ${ }^{1}$ London Centre for Nanotechnology, University College London, 17-19 Gordon Street, London WC1H OAH, \\ United Kingdom \\ ${ }^{2}$ CEA-LIST, Laboratoire de Technologie des Détecteurs, Bâtiment 451-Boîte courrier 45, \\ Centre d'Etudes de Saclay, 91191 Gif-sur-Yvette, France
}

(Received 6 September 2007; accepted 28 November 2007; published online 5 March 2008)

\begin{abstract}
The use of diamond as a semiconductor for the realization of transistor structures, which can operate at high temperatures $(>700 \mathrm{~K})$, is of increasing interest. In terms of bipolar devices, the growth of $n$-type phosphorus doped diamond is more efficient on the (111) growth plane; $p$-type boron-doped diamond growth has been most usually grown in the (100) direction and, hence, this study into the electronic properties, at high temperatures, of boron-doped diamond (111) homoepitaxial layers. It is shown that highly doped layers (hole carrier concentrations as high as $2 \times 10^{20} \mathrm{~cm}^{-3}$ ) can be produced without promoting the onset of (unwanted) hopping conduction. The persistence of valance-band conduction in these films enables relatively high mobility values to be measured $\left(\sim 20 \mathrm{~cm}^{2} / \mathrm{V} \mathrm{s}\right)$ and, intriguingly, these values are not significantly reduced at high temperatures. The layers also display very low compensation levels, a fact that may explain the high mobility values since compensation is required for hopping conduction. The results are discussed in terms of the potential of these types of layers for use with high temperature compatible diamond transistors.

(C) 2008 American Institute of Physics. [DOI: 10.1063/1.2837114]
\end{abstract}

\section{INTRODUCTION}

It has been known for sometime that boron within crystalline diamond leads to the formation of an acceptor state and, hence, $p$-type conductivity. ${ }^{1}$ An activation energy for the acceptor states of around $0.37 \mathrm{eV}$ was established. Compensation of the boron acceptors by donor levels within the crystal, most normally associated with nitrogen, was found to be growth sector dependent with $\{110\}$ directions displaying the highest number of uncompensated acceptors at low doping concentrations, while (111) suffered the least from compensation at high doping levels. ${ }^{2}$ The emergence of chemical vapor deposition (CVD) methods for the formation of diamond films led to a number of investigations of the nature of boron within CVD grown homoepitaxial, single crystal, films. ${ }^{3-5}$ These studies concentrated on (100) oriented growth and used diborane as a source of boron during the CVD process. Metallic conduction was reported for the temperature range $300-770 \mathrm{~K}$ for a boron concentration of $3 \times 10^{20} \mathrm{~cm}^{-3}{ }^{3}$ A study by Visser et al. ${ }^{6}$ reported on the influence of crystal orientation in terms of (100) and (110) samples; higher boron concentrations and lower carrier mobilities were found for the (110) samples compared with (100), for the same growth conditions, which was explained in terms of a differing growth mechanism for each. In a comprehensive study of homoepitaxial (100) films grown by microwave-plasma CVD, Borst and Weiss ${ }^{7}$ found that the activation energy for the boron-induced acceptor state varied from $0.37 \mathrm{eV}$ to effectively zero over the

\footnotetext{
a) Author to whom correspondence should be addressed. Electronic mail: r.jackman@ucl.ac.uk.
}

boron concentration range $10^{17}-3 \times 10^{20} \mathrm{~cm}^{-3}$, consistent with the expected metal-insulator (Mott) transition of 2 $\times 10^{20} \mathrm{~cm}^{-3} .8$ The influence of compensating nitrogen within CVD grown (100) diamond homoepitaxial films has been explicitly studied by Sonoda and co-workers, ${ }^{9}$ who reported a four times increase in Hall mobility values when ultrahigh purity, low nitrogen containing, growth gases were used during the CVD process. Lagrange et al. ${ }^{10}$ grew homoepitaxial (100) films with boron concentrations within the range $5 \times 10^{16}-8 \times 10^{20} \mathrm{~cm}^{-3}$. For boron concentrations up to $2 \times 10^{17} \mathrm{~cm}^{-3}$ the activation energy was observed to be the $0.37 \mathrm{eV}$ expected for the ionization of boron with compensation ratios of around $10 \%$. Above this and up to $10^{19} \mathrm{~cm}^{-3}$, an $E_{a}$ value of 0.185 was determined, with a compensation ratio less than $10 \%$, while hopping conduction was considered dominant above this, with metallic conductivity at boron concentrations above $3 \times 10^{20} \mathrm{~cm}^{-3}$.

An important development in the field of diamond electronics has been the development of $n$-type diamond homoepitaxial films through the introduction of phosphorus containing gases during the CVD process. ${ }^{11}$ However, it has now been firmly established that the phosphorus donor level lies deep within the diamond band gap at around $0.6 \mathrm{eV}$ and that the most effective creation of substitutional phosphorus requires the use of (111) crystal orientations. ${ }^{12}$ The pursuit of diamond-based bipolar devices would therefore appear to require the development of high quality boron-doped (111) films to enable the use of phosphorus doped $n$-type diamond. Moreover, the deep donor level created by phosphorus implies that the fabrication of devices for high temperature applications is likely to be the most useful avenue to explore for this form of diamond technology. Indeed, high tempera- 
TABLE I. The growth conditions used for sample types 1-5.

\begin{tabular}{lccccc}
\hline \multicolumn{1}{c}{ Sample type } & 1 & 2 & 3 & 4 & 5 \\
\hline $\mathrm{CH}_{4}$ flow (sccm) & 0.8 & 0.74 & 0.74 & 0.74 & 0.74 \\
$\mathrm{H}_{2}$ flow (sccm) & 500 & 500 & 500 & 500 & 500 \\
$\mathrm{TMB}$ flow (sccm) & 0 & 0.024 & 0.05 & 0.1 & 1.25 \\
Growth duration (hours) & 1.5 & 1.5 & 1.5 & 1.5 & 1.5 \\
Power (W) & 700 & 700 & 700 & 700 & 700 \\
Temperature $\left(+/-10^{\circ} \mathrm{C}\right)$ & 880 & 880 & 880 & 880 & 910 \\
\hline \hline
\end{tabular}

ture electronics is seen as one of the major future uses of active diamond devices. However, little has currently been reported on the electronic properties of boron-doped (111) films and insight into the behavior of doped diamond at high temperatures $(>500 \mathrm{~K})$ is limited. $\mathrm{Ri}$ and co-workers ${ }^{13}$ recently reported that the growth and doping mechanisms during homoepitaxial growth of boron-doped films on (111) diamond films by CVD methods strongly differed from those on (100). They observed an increase in Hall mobility values and a decrease in compensation ratio as the methane concentration within the CVD process was increased, the opposite observation to that for the growth of boron-doped (100) films. In the current study we have used Hall effect measurements and scanning tunneling microscopy (STM) analysis to evaluate the properties of a range of CVD grown homoepitaxial (111) orientation boron-doped diamond films within the temperature range $300-800 \mathrm{~K}$. It will be shown that high mobility valance band transport can be maintained to much higher doping levels in diamond (111) than in (100), which is highly advantageous when it comes to the design of devices based on this material.

\section{EXPERIMENTAL METHODS}

Microwave plasma-enhanced chemical vapor deposition (MWPECVD) of homoepitaxial diamond films was carried out using methane-hydrogen-trimethylborane (TMB) gas mixtures. The growth of high quality CVD diamond critically relies upon the use of ultrapure gases; in the current work $\mathrm{H}_{2}$ was generated in situ from ultrapure water by a 0.5 $\mathrm{L} / \mathrm{min} \mathrm{H}_{2}$ generator (F-DBS Strumenti Scientifici) giving nitrogen impurity levels better than $\mathrm{N} 90$. Methane was used with N55 purity $\left(99.9995 \%\right.$, Messer B50) and TMB $\left(\mathrm{C}_{3} \mathrm{H}_{9} \mathrm{~B}\right)$ supplied as a $2000 \mathrm{ppm}$ mixture in $\mathrm{N} 70 \mathrm{H}_{2}$ (99.99999\%, Messer B10) with a nitrogen level less than $1.5 \mathrm{ppm}$. HPHT prepared single crystal diamond substrates that were (111) oriented and of type $\mathrm{Ib}$ (Sumitomo) were used throughout. These were cleaned ex situ using a boiling $\mathrm{HNO}_{3}: \mathrm{H}_{2} \mathrm{SO}_{4}(1: 1)$ solution with added $\mathrm{KNO}_{3}$, which is known to leave a graphite free, oxidized diamond surface. ${ }^{14}$ Following insertion into the MWPECVD chamber samples were further cleaned using a pure hydrogen-plasma (500 sccm, $700 \mathrm{~W}, 5 \mathrm{~min}$ ) prior to growth. Table I shows the growth conditions used to generate five different types of samples, where the principal variable is the concentration of the TMB within the growth gas mixture.

Hall effect analysis was performed using a Lakeshore 7504 Hall system with an Advanced Research Systems helium atmosphere-based closed-cycle refrigerator for low tem-

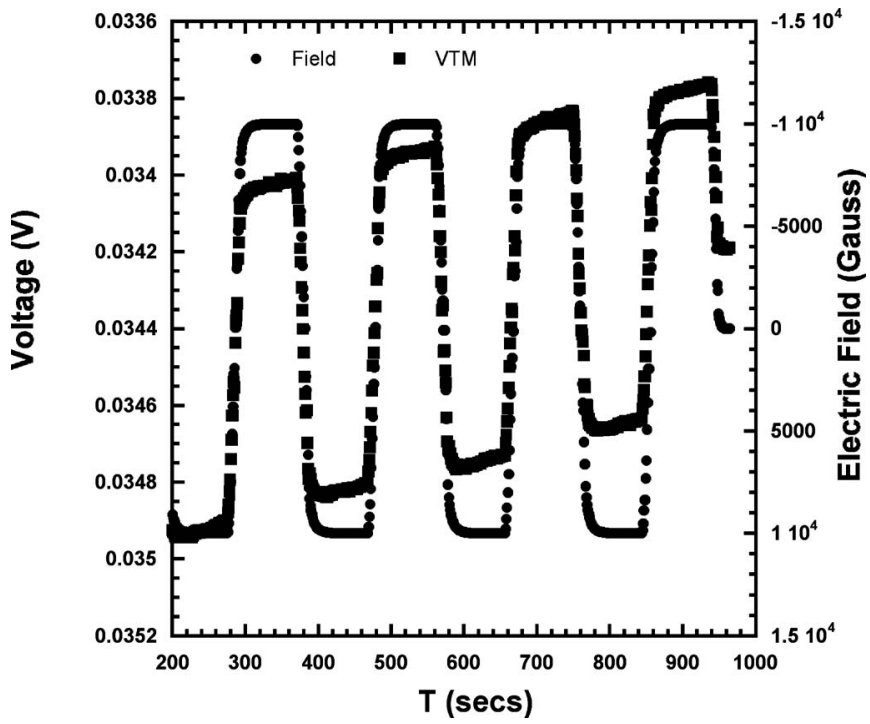

FIG. 1. Hall voltage (left scale) and electric field variation (right scale) plotted as a function of time during a Hall measurement. The stable field variation can be contrasted with the background drift that is apparent in the Hall voltage.

perature measurements (15-300 K) and a Lakeshore evacuated probe system for high temperature measurements (300$800 \mathrm{~K})$. The magnetic field was oscillated between -1 and 1 $\mathrm{T}$ as the signal was recorded. This aspect of the Hall experiments performed here is particularly important when investigating wide band gap systems, such as diamond, where significant drift in the background voltage can be encountered during detection of the Hall voltage. An example of this is shown in Fig. 1, where it can be readily seen that a single measurement of the Hall voltage could produce misleading results, as it would comprise of a voltage due to the Hall effect plus a value associated with background drift. The use of field switching method allows this problem to be overcome. STM measurements were performed using a "nanosurf" STM in air.

\section{RESULTS}

It has been widely observed that the CVD growth of diamond within a chamber that has previously been exposed to boron containing gases will always lead to some level of boron doping within the film, due to the persistence of boron within the growth environment. ${ }^{15}$ In the present case, films were grown using a flow rate of TMB, from zero up to 1.25 $\mathrm{sscm}$. All films were grown to a thickness of approximately $1 \mu \mathrm{m}$.

STM images revealed a change in surface morphology when the highest boron levels within the CVD growth gas mixture were used, but retention of the substrate surface profile until this point. For example, Fig. 2(a) shows a STM image for sample type 2, indicating a root-mean-square (rms) value of $0.2 \mathrm{~nm}$, typical of samples 1-4, while Fig. 2(b) shows an image typical for samples 5 and 6 . In this case growth domains or "hillocks" can be observed and the rms value reaches around $15 \mathrm{~nm}$. The very fact that clear STM images could be produced for all films indicates that some 


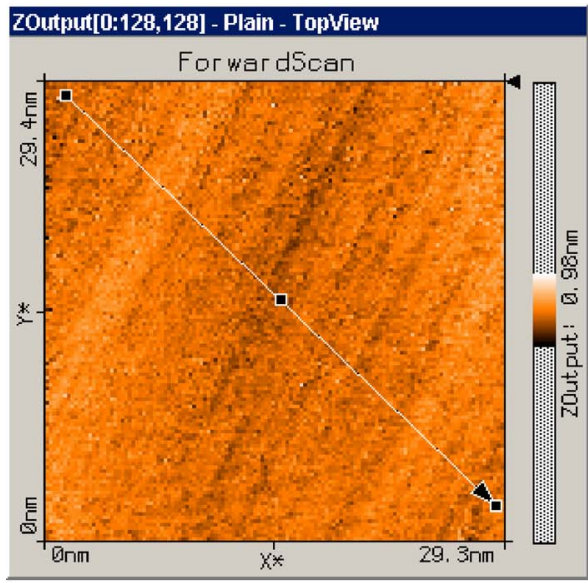

(a)

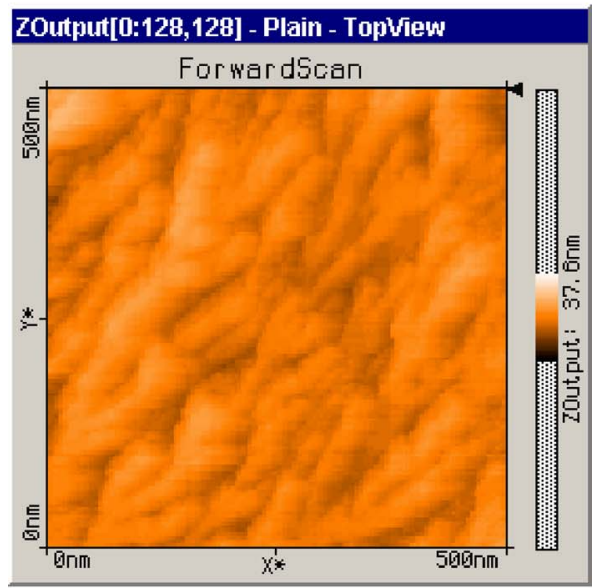

(b)

FIG. 2. (Color online) STM images for (a) sample type 2, indicating a rms value of $0.2 \mathrm{~nm}$ and (b) sample type 5 showing domains or hillocks with a rms value of around $15 \mathrm{~nm}$. electrical conductivity was present, even when films were grown without the purposeful introduction of boron containing gas to the growth mixture.

Hall effect measurements, carried out over the temperature range $300-800 \mathrm{~K}$ are presented in Fig. 3, in terms of log (sheet resistivity) versus $1 / T$ for samples $1-5$, (a)-(e). Data recorded for sample types 1-3 were qualitatively similar, showing near-linear characteristics at high temperatures, with a slightly different slope being apparent at lower temperatures. For the lightest doped sample (1), the transition is around $350 \mathrm{~K}$, where as for sample 3 it is closer to $450 \mathrm{~K}$. The resistivity values fall with sample number, i.e., $1>2$ $>3$. In the case of sample type 4 it is more difficult to distinguish the two regimes and the resistivity has decreased by a further order of magnitude. For sample type 5, the shape of the graph is completely changed, showing little resistivity variation over the entire temperature range, with the resistivity again having decreased by more than one order.

Sheet carrier concentrations are plotted in log form in Fig. 4, again against $1 / T$. For sample 1 [Fig. 4(a)], the carrier density can be seen to range from $10^{18} \mathrm{~cm}^{-3}$ at low temperatures $(300 \mathrm{~K})$ to $2 \times 10^{19} \mathrm{~cm}^{-3}$ at high temperature $(800 \mathrm{~K})$. A similar trend is evident for sample 2 [Fig. 4(b)], but the values are now $7 \times 10^{18} \mathrm{~cm}^{-3}$ to $7 \times 10^{19} \mathrm{~cm}^{-3}$. Sample 3 [Fig. 4(c)] shows some scatter within the data set, but values between $2 \times 10^{19}$ and $7 \times 10^{19} \mathrm{~cm}^{-3}$ can be determined. Interestingly, the trends observed for samples 4 and 5 differ strongly from the others. In both cases there is some scatter in the data points, but the change of carrier density with temperature is much less pronounced and lies within the range $2-4 \times 10^{19} \mathrm{~cm}^{-3}$ for sample 5 [Fig. 4(d)] to $8-30$ $\times 10^{21} \mathrm{~cm}^{-3}$ for sample 5 [Fig. 4(e)].

Figure 5 presents the mobility data as a function of temperature for the various samples. Sample 1 [Fig. 5(a)] shows an increase in mobility, peaking at $20 \mathrm{~cm}^{2} / \mathrm{V} \mathrm{s}$ at $450 \mathrm{~K}$,
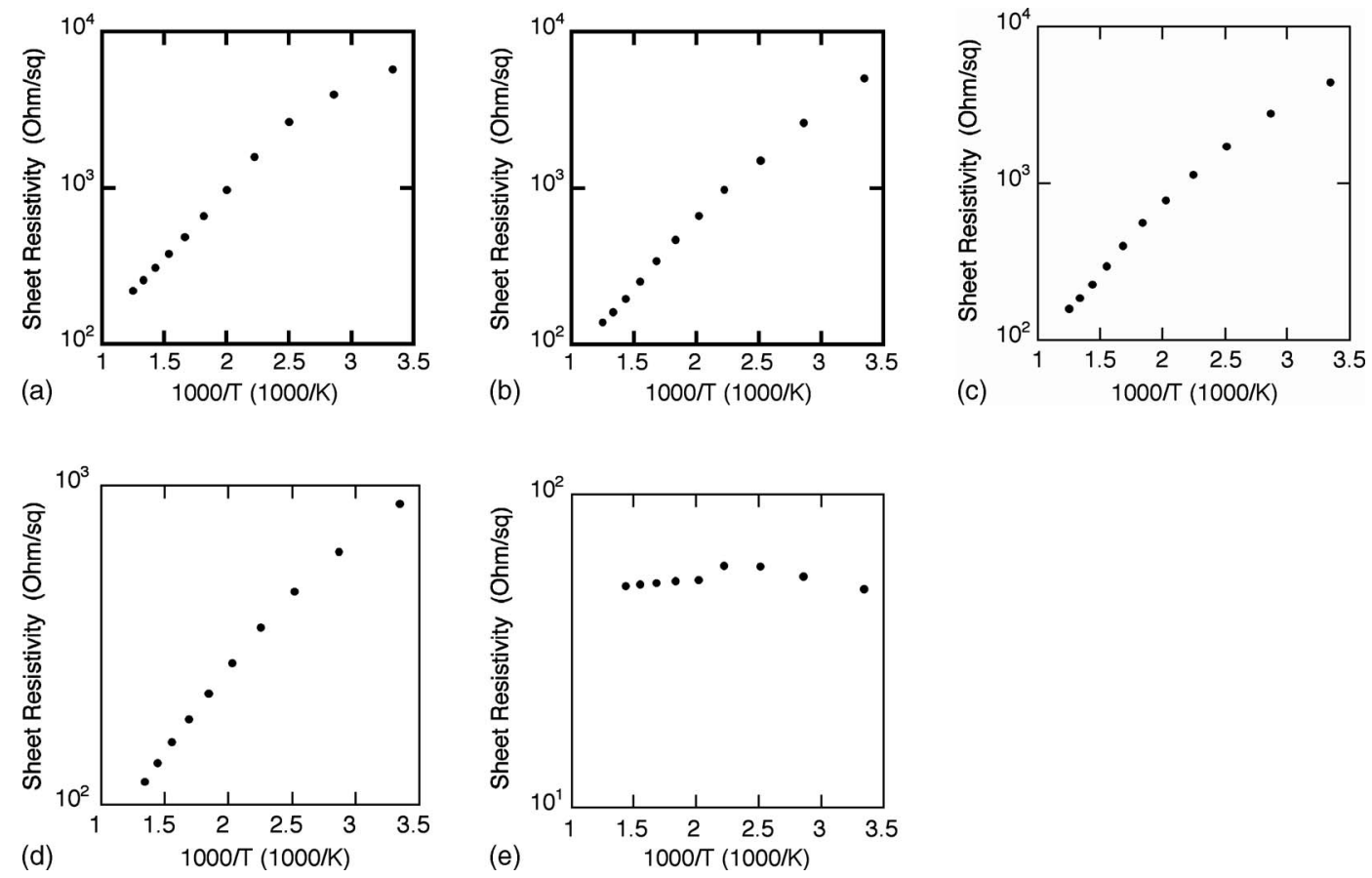

FIG. 3. $\log$ (sheet resistivity, $\Omega /$ square) vs $1 / T$ for sample types $1-5$, (a)-(e). 

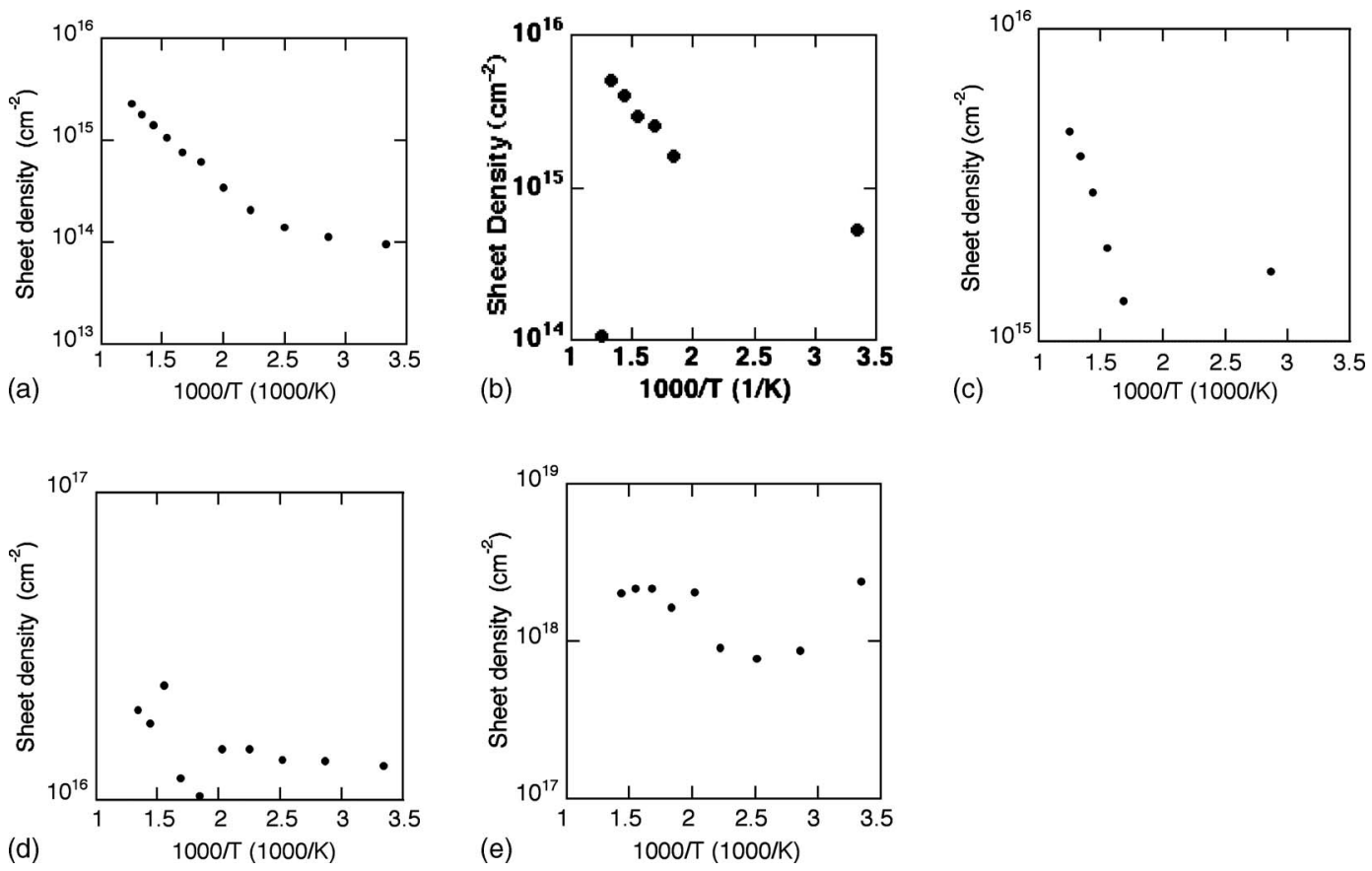

FIG. 4. Log (sheet carrier density, $\mathrm{cm}^{-2}$ ) vs $1 / T$ for sample types $1-5$, (a)-(e).

before declining to around $15 \mathrm{~cm}^{2} / \mathrm{V} \mathrm{s}$ at $800 \mathrm{~K}$. In the case of sample 2 [Fig. 5(b)], the mobility value can be seen to gradually rise from around $3 \mathrm{~cm}^{2} / \mathrm{V} \mathrm{s}$ at $300 \mathrm{~K}$ to $8 \mathrm{~cm}^{2} / \mathrm{V} \mathrm{s}$ at $800 \mathrm{~K}$, where as sample 3 [Fig. 5(c)] remains between 8 and $11 \mathrm{~cm}^{2} / \mathrm{V} \mathrm{s}$ for most of this temperature range. Sample 4 [Fig. 5(d)] shows an increase of 6 $-13 \mathrm{~cm}^{2} / \mathrm{V} \mathrm{s}$ with increasing temperature, while sample 5 [Fig. 5(e)] shows a less significant trend with much lower mobility values in the region of $0.1 \mathrm{~cm}^{2} / \mathrm{V} \mathrm{s}$.

\section{DISCUSSION}

In the current study the temperature range investigated was $300-800 \mathrm{~K}$ and samples $1-3$ were expected to have a lower doping density than samples 4 and 5 . This would indeed appear to be the case, given the variation in sheet resistivity that can be seen within Fig. 3. Thermal activation energies associated with the change in resistivity of each sample type can be determined from these plots and these are given in Table II. Borst and Weiss ${ }^{7}$ have previously identified
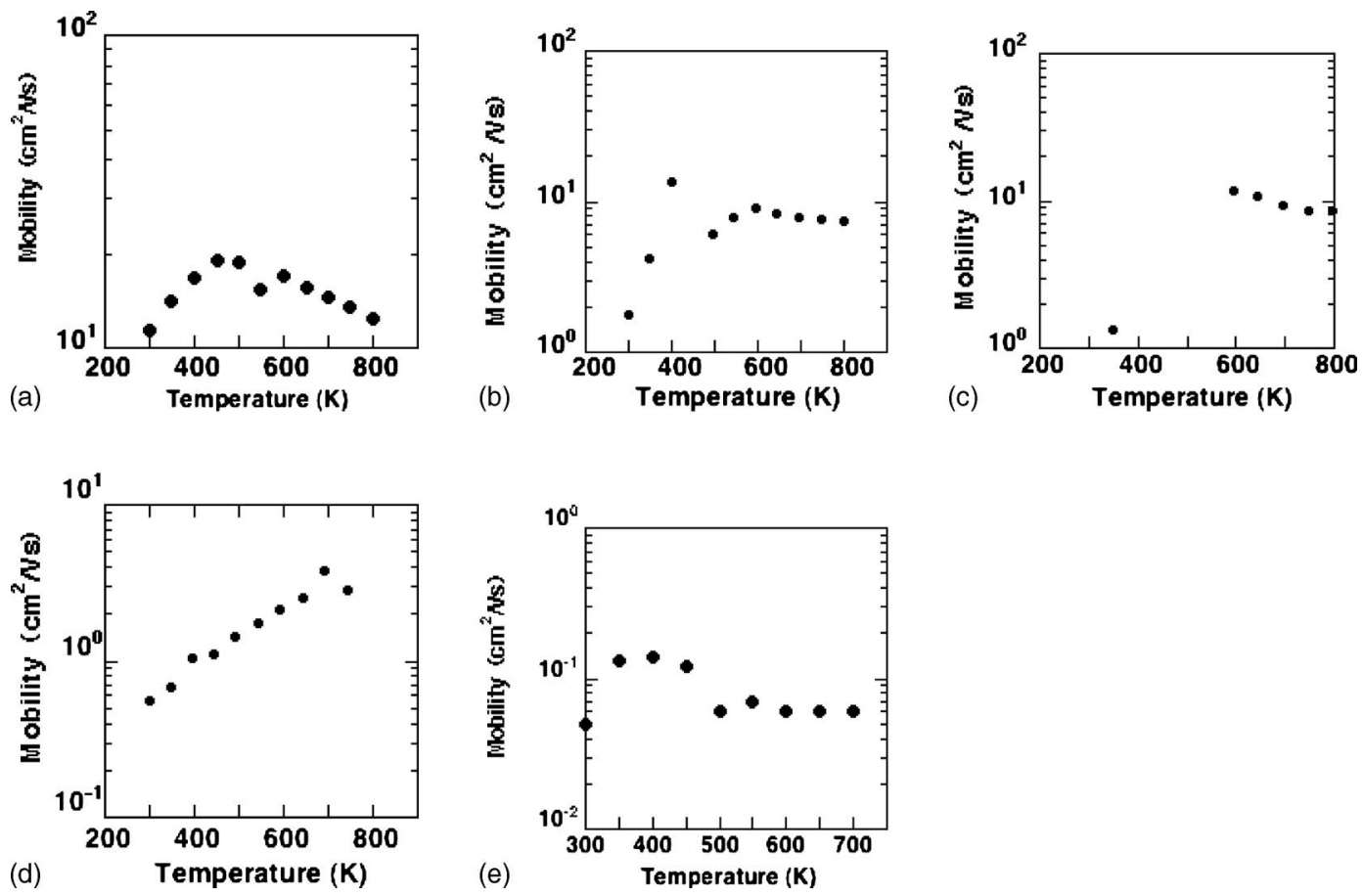

FIG. 5. Hole mobility $\left(\mathrm{cm}^{2} / \mathrm{V}\right.$ s) vs $1 / T$ for sample types $1-5$, (a)-(e). 
TABLE II. Measured activation energies for sample types $1-5$, the doping density that has previously been associated with such values for diamond (100) films (Ref. 7) and the actual value of acceptor state density $\left(N_{A}\right)$ measured in the current study.

\begin{tabular}{|c|c|c|c|c|c|c|}
\hline $\begin{array}{l}\text { Sample } \\
\text { type }\end{array}$ & $\begin{array}{l}\text { Activation } \\
\text { energy } \\
(\mathrm{eV})\end{array}$ & $\begin{array}{c}\text { Measured carrier } \\
\text { density } \\
\left(\mathrm{cm}^{-3}\right) @ 800 \mathrm{~K}\end{array}$ & $\begin{array}{c}N_{A}-N_{D} \text { by extrapolation } \\
\text { to high } T \\
\left(\mathrm{~cm}^{-3}\right)\end{array}$ & $\begin{array}{l}N_{D} \text { determined } \\
\text { by simulation }\left(\mathrm{cm}^{-3}\right)\end{array}$ & $\begin{array}{c}\text { Carrier mobility } \\
\left(\mathrm{cm}^{2} / \mathrm{V} \mathrm{s}\right) @ 800 \mathrm{~K}\end{array}$ & $\begin{array}{c}\text { Maximum carrier mobility } \\
\left(\mathrm{cm}^{2} / \mathrm{V} \mathrm{s}\right) \\
\text { (temperature, } \mathrm{K})\end{array}$ \\
\hline 1 & 0.176 & $3 \times 10^{19}$ & $6 \times 10^{20}$ & $2 \times 10^{18}$ & 15 & $20(450 \mathrm{~K})$ \\
\hline 2 & 0.170 & $7 \times 10^{19}$ & $1 \times 10^{21}$ & $3 \times 10^{18}$ & 9 & $10(600 \mathrm{~K})$ \\
\hline 3 & 0.160 & $8 \times 10^{19}$ & $2 \times 10^{21}$ & $3 \times 10^{18}$ & 9 & $10(600 \mathrm{~K})$ \\
\hline 4 & 0.095 & $2 \times 10^{20}$ & $\ldots$ & & 14 & $15(700 \mathrm{~K})$ \\
\hline 5 & 0.004 & $2 \times 10^{22}$ & $\ldots$ & & 0.07 & $0.1(400 \mathrm{~K})$ \\
\hline
\end{tabular}

a decreasing trend in the activation energy associated with boron-doped diamond films with increasing boron concentration for (100) diamond films. Their data showed that would suggest that the activation energies measured in the present arise due to very high doping levels within the diamond films.

The strong variation in the activation energy for electrical conduction within these samples implies the existence of more than one conduction mechanism. In addition to the transport of free carriers (in our case holes within the valance band), doped semiconductors can support conduction through carrier "hopping." Hopping conductivity is a conventionally associated with low temperatures in materials such as silicon, but can occur at room temperature or above in diamond, as the boron acceptor state is deep within the diamond band gap. Hopping conduction is normally restricted to samples with significant compensating defects, as donors are required to supply an electron to the boron acceptors, which are not thermally ionized at modest temperatures. A compensation ratio $K=N_{D} / N_{A}$ can be used to characterize differing samples. Charge transfer occurs by tunneling in the process of nearest neighbor hopping ${ }^{16}$ or by thermal activation in variable range hopping. ${ }^{17}$

Mamin and Inushima ${ }^{18}$ discussed the basis of conductivity in heavily boron-doped diamond films, referring to the (100) system. Under high doping conditions it was supposed that the nearest neighbor of each boron atom might no longer be carbon, with combinations such as B-B, B-C-B, B-C$\mathrm{C}-\mathrm{B}$, etc., becoming likely. The fraction of boron atoms occupying these states will depend upon both statistical laws and the nature of the growth conditions used. It can also be supposed to be affected by the crystallographic orientation of the growth plane. This kind of nontrivial state will form a shallow acceptor level with an energy less than $0.37 \mathrm{eV}$; Mamin and Inushima assumed that these states have an energy of $0.06 \mathrm{eV}$ above the valance band and that the relevant statistical law was $N_{2}$ (additional boron states) $=N_{B}^{2} \quad V_{0}$, where $V_{0}$, the volume of the interaction area was taken as $5 \mathrm{~nm}^{3}$ ). This leads to the assumption that when $N_{B}$ $=10^{19} \mathrm{~cm}^{-3}, N_{2}$ will be equal to $5 \times 10^{17} \mathrm{~cm}^{-3}$. If we further assume that the number of compensating donors, $N_{D}$, is small compared to $N_{B}$, then as the doping level increases $N_{D}$ will increase more slowly than $N_{2}$, until we reach a point where $N_{2}>N_{D}$. In this case the donors only compensate the states associated with $N_{2}$ since the energy associated with these states is so small compared to the $0.37 \mathrm{eV}$ of $N_{B}$. The conductivity behavior of the samples should now change dramatically, with the coexistence of band and hopping conductivity over a wide temperature range. It was proposed that the emergence of a range of shallow states at very high doping levels leads to conductivity that is mainly hopping in character. Langrange and co-workers ${ }^{10}$ proposed that the transition to hopping conductivity in high quality homoepitaxial (100) diamond films occurred at a boron concentration of $1 \times 10^{19} \mathrm{~cm}^{-3}$, with metallic conduction at boron levels of $>2 \times 10^{20} \mathrm{~cm}^{-3}$. Hopping conductivity is generally seen as undesirable within semiconductor systems, as it is associated with low mobility values. ${ }^{8}$

To determine the concentrations $N_{A}$ and the compensating level of $N_{D}$ numerical simulations were performed using the equation for a partially compensated semiconductor

$$
\begin{aligned}
P\left(p+N_{D}\right) /\left(N_{A}-N_{D}-p\right)= & {\left[2 \pi m_{h} k T / h^{2}\right]^{3 / 2} } \\
& \times \exp \left(-E_{A} / k T\right),
\end{aligned}
$$

where $p, m_{h}, k, T$, and $E_{A}$ are the hole concentration, the effective mass of the hole, the Boltzmann constant, sample temperature, Planck constant, and the activation energy for the ionization of the hole, respectively. ${ }^{19}$ The density difference $N_{A}-N_{D}$ can be estimated from extrapolation to the high temperature limit of the experimental $\ln p$ versus $T^{-1}$ plots. The value of $m_{h}$ can be taken to be $0.48 m_{0}$. On this basis the values of $\left(N_{A}-N_{D}\right)$ and $N_{D}$ can be seen in Table II, alongside the measured carrier concentrations at $800 \mathrm{~K}$. It is clear that the actual doping level in these (111) films is higher than that predicted from the use of activation energy data derived from measurements on (100) material. ${ }^{7}$ The compensation ratio, $N_{A} / N_{D}$, can be seen to be very low, lying in the range $0.15 \%-0.33 \%$.

It is well established in conventional semiconductor systems that ionized impurity scattering dominates observed carrier mobility values at low temperatures, while phonon scattering controls high temperature mobilities. ${ }^{16}$ When carriers are moving relatively slowly (low $T$ regime) the change in mobility is expected to follow a $T^{1.5}$ trend, while at higher temperatures, the influence of thermal acoustic phonons is likely to lead to a variation according to $T^{-1.5}$. Such classical behavior has rarely been seen in diamond films. For example, Tsubota et al. ${ }^{17}$ prepared boron-doped (100) homoepitaxial films using microwave-plasma CVD identifying a $T^{2.8}$ relationship for mobility between 200 and $300 \mathrm{~K}$, with a $T^{-2.8}$ relationship being apparent within the range 300-500 $\mathrm{K}$. While offering no explanation for these values, the authors noted the results were consistent with other studies in- 
volving both natural type IIb (boron containing) and CVD grown boron-doped homoepitaxial films. At the low temperature of $243 \mathrm{~K}$ and the extremely low hole concentration of $5 \times 10^{12} \mathrm{~cm}^{-3}$ (not useful for device applications), a Hall mobility value as high as $2020 \mathrm{~cm}^{2} / \mathrm{Vs}$ was recorded. Earlier work by Hatta, Sonoda, and Ito, ${ }^{20}$ again concentrating on homoepitaxial (100) films, found mobility to be dependent upon $T^{-(2.7-3.1)}$ within the temperature range $300-500 \mathrm{~K}$. More recently, Teraji and co-workers ${ }^{21}$ grew boron-doped (100) homoepitaxial films using a high power density microwave plasma CVD approach. The films showed very low compensation ratios (of the order of $1 \%$ ) and mobility values that varied with temperature according to $T^{-(1.3-1.6)}$ at temperatures below $270 \mathrm{~K}$, with a value of $T^{-(4.0-4.5)}$ above this temperature. These curious results were suggested to result from phonon scattering at low temperatures with the reason for the higher temperature result remaining unclear. These observations suggest diamond can behave in a manner that conflicts with established semiconductor theory. Again, high Hall mobility values $\left(2750 \mathrm{~cm}^{2} / \mathrm{V} \mathrm{s}\right)$ were reported for low temperatures $(215 \mathrm{~K})$ and very low carrier concentrations $\left(3 \times 10^{14} \mathrm{~cm}^{-3}\right)$. Wang et al. ${ }^{22}$ reported a $T^{-3.9}$ dependence on mobility for the entire temperature range $300-800 \mathrm{~K}$ for (100) homoepitaxial films with an acceptor concentration of $1 \times 10^{19} \mathrm{~cm}^{-3}\left(N_{D} \sim 10^{17} \mathrm{~cm}^{-3}\right)$ and a room temperature Hall mobility of $910 \mathrm{~cm}^{2} / \mathrm{V} \mathrm{s}$. This discussion highlights the difficulty in considering the origin of carrier scattering at differing temperatures and different doping regimes, even within the relatively well-studied diamond (100) borondoped system. In terms of the (111) system, little is currently known, with $\mathrm{Gi}$ and co-workers ${ }^{13}$ confining their observations to the temperature range $200-450 \mathrm{~K}$ and making no comment on the variation of mobility with temperature. They reported a room temperature mobility value of $364 \mathrm{~cm}^{2} / \mathrm{V} \mathrm{s}$ for the very low carrier concentration of $2.9 \times 10^{13} \mathrm{~cm}^{-3}$ (again not useful for devices). The compensation ratios determined were high at $3 \%-28 \%$.

In the current case, low compensation ratios have been determined, alongside carrier concentrations and relatively high mobility values, at high temperatures (800 K). For example, in the case of sample type 1, (Table II) the value for hole mobility peaks at $\sim 20 \mathrm{~cm}^{2} / \mathrm{V} \mathrm{s}(450 \mathrm{~K})$ at the (high) carrier concentration of $3 \times 10^{19} \mathrm{~cm}^{-3}$, and only falls to $15 \mathrm{~cm}^{2} / \mathrm{V} \mathrm{s}$ at $800 \mathrm{~K}$, where phonon scattering is expected to reduce the mobility value. Moreover, the lower temperature variation of mobility as $T^{1.3}$ and the higher temperature variation being as $T^{-0.9}$ in this sample is indicative of nearclassical conduction with the diamond semiconductor. In sample type 4 , the $800 \mathrm{~K}$ mobility value is rather similar to sample type $1\left(14 \mathrm{~cm}^{2} / \mathrm{V} \mathrm{s}\right)$, despite the increase in carrier concentration to $2 \times 10^{20} \mathrm{~cm}^{-3}$, and the peak in mobility value is now at $700 \mathrm{~K}$ rather than $450 \mathrm{~K}$. Indeed, a trend in increasing the temperature for the peak in mobility value is evident through sample types $1-4$. This result implies a decrease in the relative effect of phonon scattering compared to ionized impurity scattering as the dopant concentration increases. This is not surprising in itself, but it is surprising that the mobility values remain relatively high for all of these sample types. Figure 6 shows a plot of the mobility data

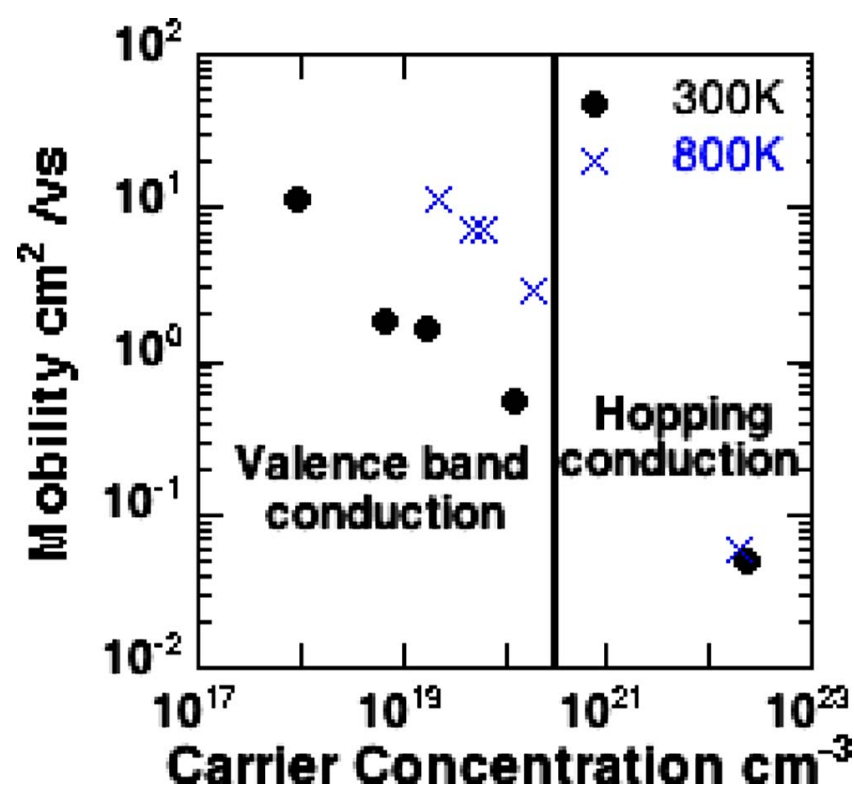

FIG. 6. (Color online) Hole mobility $\left(\mathrm{cm}^{2} / \mathrm{V} \mathrm{s}\right)$ plotted against carrier density $\left(\mathrm{cm}^{-3}\right)$ for the samples grown here, measured at 300 and $800 \mathrm{~K}$.

measured here, against the sheet carrier density for two temperatures (300 and $800 \mathrm{~K}$ ), clearly revealing the hopping and valance conduction regions observed. This observation is very encouraging for the high temperature operation of diamond devices based around highly boron-doped (111) layers, as device operation will not be degraded as severely by high temperatures as would have previously been expected. It can be speculated that the very low compensation levels achieved in here are indicative of the very high quality homoepitaxial process that has been achieved. Since compensation is required for hopping conductivity to occur, this result is likely to be the reason that high mobility (valance band conduction) is maintained in sample types 4 ; in studies of boron-doped diamond (100) the doping level evident in sample type 4 would have already led to the loss of valance band-conduction and the onset of the very low mobility values associated with hopping. In the present case it is only sample type $5\left(2 \times 10^{22} \mathrm{~cm}^{-3}\right.$ holes, Table II $)$ that displays the low mobility values associated with carrier hopping $\left(<0.1 \mathrm{~cm}^{2} / \mathrm{V} \mathrm{s}\right)$.

\section{CONCLUDING REMARKS}

High quality homoepitaxial films have been grown on type $1 \mathrm{~b}$ (111) single crystal diamonds. TMB has been used to introduce boron as a substitutional dopant species during the homoepitaxial growth process, with free carriers (holes) concentrations as high as $2 \times 10^{20} \mathrm{~cm}^{-3}$ while maintaining (valance) band conduction displaying mobilties of the order of $15 \mathrm{~cm}^{2} / \mathrm{V} \mathrm{s}$. These high values are thought to arise through delaying the onset of hopping conductivity within these films, due to the extremely low compensation levels observed for these films. Only at carrier concentrations measured as high as $2 \times 10^{22} \mathrm{~cm}^{-3}$ does the mobility collapse to values associated with hopping $\left(<0.1 \mathrm{~cm}^{2} / \mathrm{V} \mathrm{s}\right)$. Interestingly, the mobility values in these films are not strongly degraded by increasing temperatures, with the maximum val- 
ues of mobility in some films actually occurring at $700 \mathrm{~K}$. These results are very promising for the potential application of highly boron-doped (111) homoepitaxial diamond layers for high temperature devices applications. Further studies into the influence of substrate surface preparation on the electronic characteristics of the subsequently grown epilayers are currently underway, but it is clear that the ultrapure gases used for growth in the current instance and the growth parameters used can lead to very high quality-doped diamond. The integration of these types of layers with more lightly phosphorus-doped diamond (111) epitaxial layers can be seen to be an essential step toward the realizing of one sidedabrupt junction bipolar devices from diamond for high temperature device applications.

\section{ACKNOWLEDGMENTS}

This work has been partially funded by the Engineering and Physical Sciences Research Council (U.K.) (EPSRCGB) under Award No. EP/D029651/1, "Diamond: A semiconductor for power devices for high temperature aerospace applications," a program which also receives support from Rolls-Royce plc; Element Six, Ltd., and Cambridge Semiconductors, Ltd. All are gratefully acknowledged.

${ }^{1}$ A. T. Collins and A. W. S. Williams, J. Phys. C 4, 1789 (1971).

${ }^{2}$ R. C. Burns, V. Cvetkovic, C. V. Dodge, D. J. F. Evans, M. L. T. Rooney, P. M. Spear, and C. M. Welbourn, J. Cryst. Growth 104, 257 (1990).

${ }^{3}$ N. Fujimori, H. Nakahata, and T. Imai, Jpn. J. Appl. Phys., Part 1 29, 824
(1990).

${ }^{4}$ H. Shiomi, Y. Nishibayashi, and N. Fujimori, Jpn. J. Appl. Phys., Part 1 30, 1363 (1991).

${ }^{5}$ S. A. Grot, C. W. Hatfield, G. S. Gildenblat, A. R. Badzian, and T. Badzian, Appl. Phys. Lett. 58, 1542 (1991).

${ }^{6}$ E. P. Visser, G. J. Bauhuis, G. Janssen, W. Vollenberg, W. J. P. Van Enckevort, and L. J. Giling, J. Phys.: Condens. Matter 4, 7365 (1992).

${ }^{7}$ T. H. Borst and O. Weis, Phys. Status Solidi A 154, 423 (1996).

${ }^{8}$ B. I. Shklovskii and A. L. Efros, in Electronic Properties of Doped Semiconductors, edited by M. Cardona (Springer, Berlin, 1984).

${ }^{9}$ S. Sonoda, J. H. Won, H. Yagi, A. Hatta, T. Ito, and A. Hiraki, Appl. Phys. Lett. 70, 2574 (1997).

${ }^{10}$ J. P. Lagrange, A. Deneuville, and E. Gheeraert, Carbon 37, 807 (1999).

${ }^{11}$ S. Koizumi, H. Ozaki, M. Kamo, Y. Sato, and T. Inuzuka, Appl. Phys. Lett. 71, 1065 (1997).

${ }^{12}$ M. Katagiri, J. Isoya, S. Koizumi, and H. Kanda, Appl. Phys. Lett. 85, 6365 (2004)

${ }^{13}$ S. G. Ri, H. Kato, M. Ogura, H. Watanabe, T. Makino, S. Yamasaki, and H. Okushi, Diamond Relat. Mater. 14, 1964 (2005).

${ }^{14}$ B. Baral, S. S. M. Chan, and R. B. Jackman, J. Vac. Sci. Technol. A 14, 2303 (1996)

${ }^{15}$ A. Tajani, C. Tavares, M. Wade, C. Baron, E. Gheeraert, E. Bustarret, S. Koizumi, and D. Araujo, Phys. Status Solidi A 201, 2462 (2004).

${ }^{16}$ A. Miller and E. Abrahams, Phys. Rev. 120, 745 (1960).

${ }^{17}$ T. Tsubota, T. Fukui, T. Saito, K. Kusakabe, S. Morooka, and H. Maeda, Diamond Relat. Mater. 9, 1362 (2000).

${ }^{18}$ R. F. Mamin and T. Inushima, Phys. Rev. B 63, 033201 (2001).

${ }^{19}$ C. Wang, M. Irie, K. Kimura, T. Teraji, and T. Ito, Jpn. J. Appl. Phys., Part 1 40, 4145 (2001)

${ }^{20}$ A. Hatta, S. Sonoda, and T. Ito, Diamond Relat. Mater. 8, 1470 (1999).

${ }^{21}$ T. Teraji, H. Wada, M. Yamamoto, K. Arima, and T. Ito, Diamond Relat. Mater. 15, 602 (2006)

${ }^{22}$ C. Wang, M. Irie, K. Kimura, T. Teraji, and T. Ito, Jpn. J. Appl. Phys., Part 1 40, 4145 (2001). 